\title{
A SIMULATION STUDY TO REDUCE NURSE OVERTIME AND IMPROVE PATIENT FLOW TIME AT A HOSPITAL ENDOSCOPY UNIT
}

\author{
Javad Taheri \\ North Carolina State University \\ Raleigh, NC 27695, USA
}

\author{
Ziad Gellad \\ Dariele Burchfield \\ Kevin Cooper \\ Duke University Medical Center \\ Durham, NC 27710, USA
}

\begin{abstract}
Increasing demand for endoscopic procedures, coupled with decreasing insurance reimbursement, has necessitated improvement in endoscopy unit operational performance measures, such as increasing throughput and reducing staff overtime without an increase in patient waiting time. In pursuit of improving these measurements, maintaining the nurse-to-patient ratio requirements in the recovery area throughout the clinic's operation time is a challenging problem for endoscopy units. To maintain compliance with this ratio, patients occasionally have to be held in the procedure rooms during the clinic's peak time. On the other hand, level loading could potentially increase the amount of overtime. In this paper, we describe our efforts to use discrete event simulation to investigate the impact of several strategies to address the minimum recovery nurse requirements in the endoscopy unit of Duke University Medical Center. Our objective was to minimize patient flow times and nurse overtime while sustaining the required nursepatient staffing ratio in recovery.
\end{abstract}

\section{INTRODUCTION}

Increasing demand for endoscopic procedures, coupled with decreasing insurance reimbursement, has necessitated improvement in endoscopy unit efficiency. Traditional methods to improve the efficiency of clinical operations at large academic health centers have had limited success due to the complexity of these health centers. Furthermore, as described in the Institute of Medicine and National Academy of Engineering's report on Systems Engineering in Health Care, very little talent has been focused on improving or optimizing the productivity of the health care system. (Reid et al. 2005) A renewed focus on value in health care thus offers an opportunity to promote systems engineering methodology to improve health care efficiency.

In this paper, we describe our efforts partnering with the Duke University Medical Center (DUMC) endoscopy unit to improve the efficiency of clinical operations in the unit. Our goal was to create a discrete event simulation model of the endoscopy unit and then use this model to recommend changes in staff configuration and patient scheduling to minimize nurse overtime hours. In this paper, we summarize our review of the literature, provide an introduction to the DUMC endoscopy unit, and discuss in detail 
the creation of the discrete event simulation model. We then review our results, including scenario analyses that resulted in actionable changes within the endoscopy unit.

\section{BACKGROUND}

\subsection{Overview of the DUMC Endoscopy Unit}

The DUMC gastrointestinal endoscopy unit is a hospital-based endoscopy unit with eight procedure rooms, eight preparation bays and twelve recovery bays. The unit primarily serves outpatients although procedures are occasionally performed on inpatients when needed. In addition, inpatients from neighboring hospitals are rarely transported by ambulance to DUMC for a particular procedure and are transported back afterwards.

\subsection{Endoscopic Procedures}

About 200 procedures are performed per week. These are categorized for the purposes of our study as: general gastrointestinal procedures, including colonoscopy, sigmoidoscopy, small bowel enteroscopy, and esophagogastroduodenoscopy (EGD); endoscopic ultrasound (EUS); endoscopic retrograde cholangiopancreaticography (ERCP); and bronchoscopy. Figure 1 shows the breakdown of the procedure volume. EUS, ERCP and bronchoscopy are performed in dedicated procedure rooms, while general procedures can occur in any of the remaining five rooms.

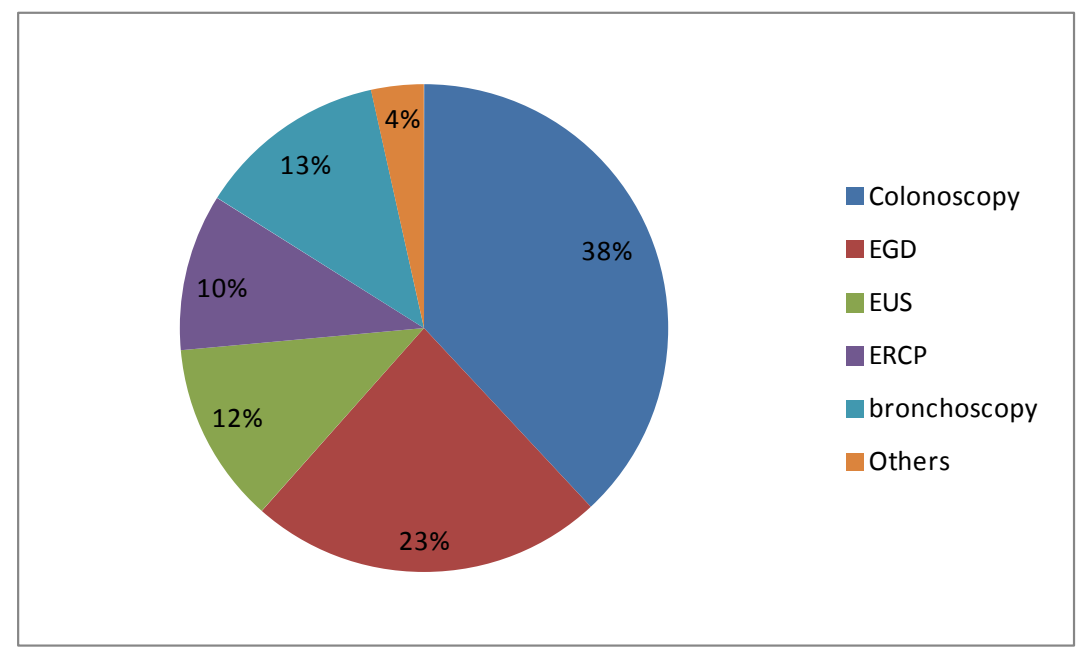

Figure 1: Proportion of procedures performed in the DUMC endoscopy unit

Procedural sedation is primarily provided by nurses who administer conscious sedation with fentanyl and midazolam under the supervision of the physician endoscopist. However, monitored anesthesia care (MAC) using nurse anesthetists is also provided for a number of procedures, including EUS, ERCP, and select general cases.

\subsection{Patient and Provider Scheduling}

On arriving in the morning, physicians are assigned room(s) which they keep for their entire shift. On certain days of the week, one physician performing general gastrointestinal procedures may be assigned two rooms for the day, while the other physicians are each assigned one. Physicians work in half-day blocks but may work an entire day depending on the day of the week. 
Time slot availability for procedures varies by procedure type and is also dependent on physician availability. Typically general gastrointestinal and bronchoscopy procedures are scheduled in 45-minute intervals between 8am and 11:45am and again from 1-3:15pm. EUS and ERCP are scheduled in 1-hour intervals between $8 \mathrm{am}$ and 11 am and again from 1-3pm. Urgent inpatient procedures are occasionally added to the schedule on the day prior to the procedure.

Nurses are scheduled in 10-hour shifts beginning at 7:30AM. At the time of the study, the unit averaged over 200 hours per month in overtime, accounting for 1.15 Full-Time Equivalents (FTE).

\subsection{Patient and Work Flow}

Figure 2 depicts the patient flow and workflow in the DUMC endoscopy unit. Patients arrive at the clinic following an appointment system. Patients check in at reception and wait in the waiting room to be called in by the nurse. Patients are required to have a driver with them. When a preparation (prep) bay is available, a nurse picks up the paperwork and summons the patient. The nurse meets the driver and informs him/her about the expected discharge time.

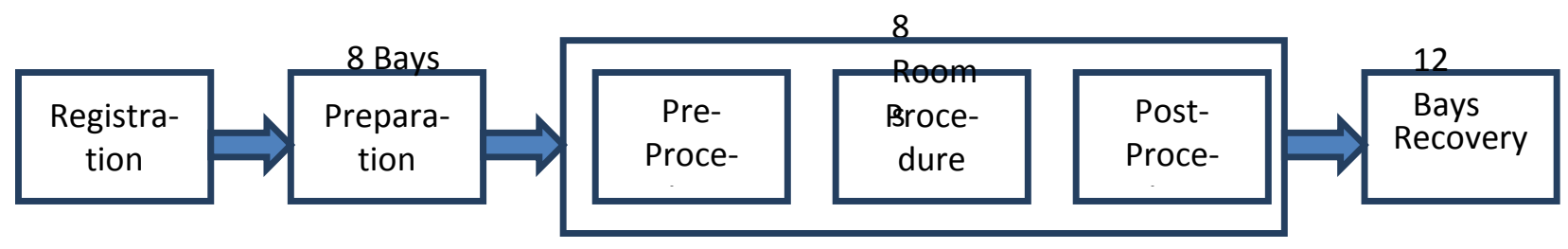

Figure 2: Patient Flow and Workflow in the DUMC Endoscopy Unit

The prep nurse then prepares the patient for the procedure in one of the 8 prep bays. Consent is also obtained by the nurses for diagnostic procedures and then reviewed by the physician prior to the procedure. While patients are waiting in a prep bay for a procedure room to become available after they have been prepared, they do not need to be monitored by a nurse. When a procedure room is available, a procedure nurse comes to the prep area and wheels the patient on a stretcher to the procedure room. When the procedure is completed, the procedure nurse wheels the patient to one of the 12 recovery bays that are distinct from the prep bays. After signing over the care of the patient to one of the recovery nurses, the procedure nurse returns to the procedure room to help turn the room over for the next procedure. The patient remains in recovery until ready for discharge. Discharge can occur when three criteria are met: 1) the patient is clinically recovered from anesthesia; 2) the physician has spoken to the patient about the results of the procedure; and 3) the patient's driver is available to assume responsibility for the patient. If any of these three criteria are not met, the patient remains in recovery.

\section{LITERATURE REVIEW}

\subsection{The Rising Demand For Endoscopic Procedure}

Colorectal cancer is the fourth leading cause of cancer death in the United States. The use of colonoscopy for screening is increasing in frequency (Harewood et al. 2004, Lieberman et al. 2005) and has become the dominant screening modality in the United States Medicare population (Schenck et al. 2009). Current endoscopic capacity is insufficient to optimally provide CRC screening to the unscreened population of the United States (Seef et al. 2004, Gellad \& Provenzale 2008), and thus health systems are struggling to meet the demand for endoscopic screening. At the same time, fiscal constraints are limiting health sys- 
tems' ability to hire additional staff to meet this increased demand. As a result, nurse overtime demands have increased.

Furthermore, gastrointestinal endoscopic procedures as a whole make up the largest percentage (32.7\%) of ambulatory surgical center claims in Medicare, (U.S. Department of Health and Human Services Report to Congress, 2011) and so significant pressures exist to decrease the costs of endoscopic practice. One mechanism used by insurers to decrease costs is to pursue value-based purchasing of endoscopy services (Deas 2006, Hewett 2010, Kim 2011). While endoscopic practices need to demonstrate value through quality, decreasing costs through improved efficiency has also become critically important.

\subsection{Application Of Simulation To Improve Efficiency Of Colonoscopy/Endoscopy Suites}

Despite the advantages of discrete event simulation (DES), its use in endoscopy units is limited. Rex et al. (2005) utilized DES to study the impact of running two rooms per endoscopy physician. They report in abstract form that although the number of procedures per physician would improve by $23 \%$, several other important metrics would suffer as the bottleneck shifts to the other resources. For example, length of stay would increase by $23 \%$, the other staff utilization would drop by $24 \%-41 \%$ and total facility operation time would increase by $7 \%$. It is not clear if this analysis modeled the impact of other critical resources, such as the pre-procedure and procedure nurses, and preparation and recovery rooms.

Berg et al. (2009) also used a patient flow simulation model to study the efficiency of an endoscopy suite. Unlike Rex et.al, their model explicitly considers the impact of resources other than the procedure rooms. The authors used data from a computerized colonoscopy database to develop their model. However, it is not clear if the data elements were detailed enough to accurately represent the dynamic behavior of the system. Furthermore, their analysis was limited to only colonoscopy procedures and thus may not be applicable to endoscopy units that perform additional procedures.

Finally, Centeno et al. (2010) also used DES modeling to study the causes of low throughput in a community endoscopy center. Their analysis attributed the low throughput to two major reasons. 1) patients were arriving too early and thus disrupted the scheduled order for patients and 2) physicians were asking to use their preferred procedure rooms. Our analysis extends this work in several notable ways: First, our analysis is focused on improving throughput by maximizing use of existing staff rather than through the addition of additional nurses. Secondly, the endoscopy unit at DUMC performs a number of procedures not discussed in the analysis of Centeno and colleagues, including ERCP, EUS and Bronchoscopy. Thirdly, the endoscopy unit at DUMC employs both anesthesiologists as well as nurses to deliver sedation to patients while the endoscopy unit analyzed by Centeno et al. uses only anesthesiologists for sedation. This added complexity allows comparisons of different sedation paradigms and an assessment of the impact of nurse-patient ratios on unit flow. Finally, the results of experiments in our analysis are described individually as opposed to fractionally as done by Centeno et al. This added detail may highlight additional opportunities for improvement, especially in regard to improving nurse staffing.

\section{SIMULATION MODEL}

\subsection{The Baseline Model}

A discrete event simulation model of patient flow in the DUMC endoscopy unit was developed using Simio (Kelton et al. 2010). Figure 3 depicts a snapshot of the animation which is a scaled representation of the DUMC endoscopy unit. The flow of patients is organized in three phases: preparation, procedure and recovery. The preparation bays are staffed by four preparation nurses. From 11:00 AM to 1:00 PM, two of these nurses take a break for lunch and provide lunch relief to other nurses. The procedure rooms and recovery area are not similarly shut down during lunch time. To aid with verification and validation 
of the model, Simio's Status Labels were used to display entities' and resources' attributes. For example, in Figure 3, the label associated with the physicians are used to verify that physicians attend the correct room and treat the correct patient. The label associated with patients included two digits, the first digit is the room number and the second digit is the arrival sequence which is used to verify that each patient is following a correct route.

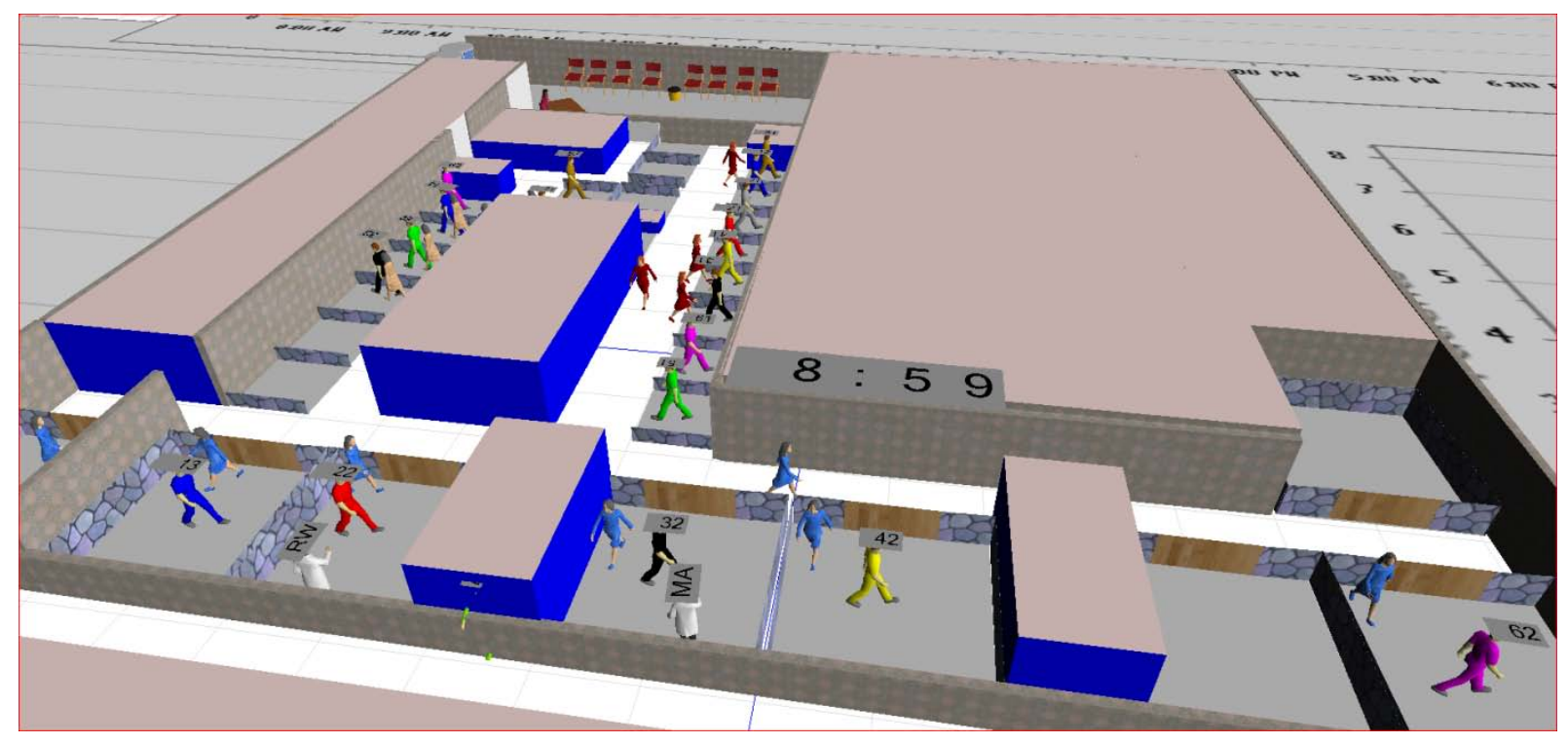

Figure 3: Screenshot of the animated simulation model

In the procedure room, three activities were modeled: pre-procedure; procedure, and post-procedure. Each procedure room is staffed by a nurse and a technician. The procedure nurse is required for all three activities. S/he is also responsible for picking up the patient from preparation and dropping the patient off in recovery. The technician was not explicitly modeled as we assumed that the technician is always available and there is sufficient time to turn the room over after the completion of the procedure. A physician is required for each procedure. At the completion of the procedure, the physician returns to his/her station to write the procedure report and prepare for the next case. A physician may use two rooms in parallel. In recovery, there are 12 bays staffed by 4 recovery nurses. The procedure nurse hands off the patient at the recovery bay to a recovery nurse. Once a patient is recovered, the patient's physician meets with the patient and discharges him/her. The recovery nurse then assists and escorts the patient to the exit.

Typically, an average of 40 appointments is scheduled; for the baseline model we chose a specific Wednesday's appointment schedule that included 41 patients. Table 1 summarizes the number of appointments for each room. On this day, room 1 was used only a half-day for bronchoscopy and rooms 2 , 3 , and 7 were sporadically used for colonoscopy and EGD. Rooms 4 and 5 were used by a single physician. Patients in rooms 1, 2, 4 and 5 were all sedated by nurses while patients in the other 3 rooms underwent monitored anesthesia care (MAC). The distinction between nurse-sedated and MAC cases is important because the sedation policy is set to change. In the baseline model, as has been practiced in the clinic so far, we assumed a nurse-patient ratio of 1 to 3 for all patients in recovery. With four recovery nurses, exceeding the nurse-patient ratio will never be any issue. While the required nurse-patient ratio for the nurse-sedated patients in recovery will continue to be 1 to 3 , it will change to 1 to 2 for patients 
undergoing MAC. This implies that the binding constraint for the recovery area becomes the nursepatient ratio.

Table 1: Number of completed patients and procedure type for the base model. MAC refers to Monitored Anesthesia Care

\begin{tabular}{|c|c|c|c|}
\hline Room & Procedure & Number of Appointments & Anesthesia \\
\hline 1 & Bronchoscopy & 4 & Nurse \\
\hline 2 & Colonoscopy/EGD & 3 & Nurse \\
\hline 3 & Colonoscopy/EGD & 4 & MAC \\
\hline 4 & Colonoscopy/EGD & 7 & Nurse \\
\hline 5 & Colonoscopy/EGD & 6 & Nurse \\
\hline 6 & EUS & 8 & MAC \\
\hline 7 & Colonoscopy/EGD & 2 & Nurse \\
\hline 8 & ERCP & 7 & MAC \\
\hline
\end{tabular}

Using existing historical timestamp data collected by the nursing staff in Provation ${ }^{\circledR}$ MultiCaregiver (WoltersKluwer Health), and Arena Input Analyzer [Kelton et al, 2009], probability distributions were estimated for all pertinent activities for each group of procedures. Table 2 depicts the probability distributions for colonoscopy and EGD where physicians are assigned a single room and when they are assigned two rooms in parallel. Table 3 depicts the probability distributions for other procedures where physicians performing bronchoscopy, EUS and ERCP procedures will always use a single room. As is expected, on average, the pre-procedure time for double room cases is longer than the single room case. However, the pre-procedure time is an overestimation of the time that it takes to prepare the patient for the procedure in the room because it includes other activities such as the waiting time for physician, time for obtaining consents, and other miscellaneous items.

Table 2: Probability Distributions for Colonoscopy and EGD Procedures (minutes)

\begin{tabular}{|l|c|c|c|c|}
\hline \multirow{2}{*}{ Activity } & \multicolumn{2}{c|}{ Single Room } & \multicolumn{2}{c|}{ Double Room } \\
\cline { 2 - 5 } & Colonoscopy & EGD & Colonoscopy & EGD \\
\hline Preparation & $70 \operatorname{BETA}(1.39,5.46)+4$ & $52 \operatorname{BETA}(1.16,3.91)+6$ & $\operatorname{GAMMA}(5.43,3.03)$ & $\mathrm{GAMMA}(9.6,1.98)$ \\
\hline Pre-Procedure & $\operatorname{GAMMA}(15.9,1.53)+2$ & $\operatorname{GAMMA}(13.7,1.56)+3$ & $\operatorname{GAMMA}(14.2,2.04)+4$ & $\operatorname{TRIANGULAR}(4,16.6,63)$ \\
\hline Procedure & $81 \operatorname{BETA}(3.25,7.34)+2$ & $\operatorname{GAMMA}(5.24,1.83)+2$ & $\mathrm{GAMMA}(3.81,5.44)+3$ & $14 \operatorname{BETA}(0.993,1.56)+1$ \\
\hline Post Procedure & $\operatorname{GAMMA}(2.57,2.22)+3$ & $\operatorname{GAMMA}(3.02,2.43)+3$ & $\mathrm{GAMMA}(1.94,2.97)+3$ & $\mathrm{GAMMA}(1.94,2.32)+5$ \\
\hline Recovery & $\operatorname{GAMMA}(13,2.23)+15$ & $\operatorname{GAMMA}(14.9,2.35)+15$ & $\operatorname{GAMMA}(10.4,2.57)+16$ & $\operatorname{TRIANGULAR}(24,33.7,80)$ \\
\hline Post Recovery & $\operatorname{GAMMA}(30.5,0.322)$ & $\operatorname{GAMMA}(30.2, .234)$ & $\operatorname{GAMMA}(22.7, .298)$ & $\operatorname{EXPONENTIAL}(6.32)$ \\
\hline
\end{tabular}


Table 3: Probability Distributions for EUS, ERCP and Bronchoscopy Procedures (minutes)

\begin{tabular}{|l|c|c|c|}
\hline \multicolumn{1}{|c|}{ Activity } & EUS & ERCP & Bronchoscopy \\
\hline Preparation & GAMMA $(6.42,3.11)$ & GAMMA $(9.97,1.64)+7$ & $\operatorname{GAMMA}(5.95,2.89)+2$ \\
\hline Pre-Procedure & GAMMA $(8.32,1.47)+3$ & $\operatorname{GAMMA}(4.55,3.73)+1$ & $\operatorname{GAMMA}(4.86,2.86)+3$ \\
\hline Procedure & GAMMA $(5.24,1.83)+2$ & $\operatorname{GAMMA}(12.6,2.96)+1$ & $\operatorname{GAMMA}(3.08,3.75)+1$ \\
\hline Post Procedure & GAMMA $(14.8,1.75)+4$ & $\operatorname{GAMMA}(3.15,3.69)+3$ & $\operatorname{GAMMA}(2.28,4.71)+1$ \\
\hline Recovery & GAMMA $(18.6,2.68)+16$ & $\operatorname{GAMMA}(49.3,1.26)+43$ & $\operatorname{GAMMA}(10.2,4.65)+18$ \\
\hline Post Recovery & GAMMA $(37.9,0.309)$ & GAMMA $(58.9, .235)$ & EXPONENTIAL $(5.03)$ \\
\hline
\end{tabular}

To calibrate the baseline model, we compared the timestamps for when the patient was ready to be moved to the procedure room as predicted by the model and the actual time the patient was moved to the room. We then kept the procedure room artificially busy for a period of time before making it available for the next patient. We considered this as idle time in the reported percent room utilization.

\subsection{Key Performance Measures}

To study the impact of the nurse-patient ratio and compare various strategies to reduce the blocking of the procedure rooms, we collected a few key performance measures: 1) Patient Flow Time is the length of time from when the patient is called in by the preparation nurse to the time that patient leaves the unit; 2) Waiting for Procedure Room is the length of time from the completion of the preparation to the time the patient leaves the prep bay for the procedure room; 3) Waiting for Recovery Nurse is the length of time from when the patient is ready to be moved to the recovery to the time a recovery nurse is available and is assigned to the patient; 4) Overtime (nurse-hour) is the total hours of recovery nurse needed from 5:00pm to the time the last patient leaves the unit.

Tables 4 and 5 provide a summary of the results from a 100 replications of the baseline model. As the results in Table 4 show, at the current nurse-patient ratio, patients are not held in the procedure room awaiting a recovery nurse. Note that room utilization is calculated for the period from 7:30am to 5:00pm and room 1 was only scheduled for a half day and rooms 2,3 , and 7 were sporadically used.

Table 4: Key Statistics for the baseline model

\begin{tabular}{|l|c|c|}
\hline \multicolumn{1}{|c|}{ Measurement } & Average & Std Deviation \\
\hline Patient Flow Time (Minutes) & 177.8 & 7.0 \\
\hline Waiting for Procedure Room (Minutes) & 31.4 & 0.4 \\
\hline Waiting for Recovery Nurse (Minutes) & 0.0 & 0.0 \\
\hline Overtime (hours) & 3.4 & 0.4 \\
\hline
\end{tabular}

Table 5: Room Utilization (\%)

\begin{tabular}{|c|c|c|}
\hline Room & Average & Std Deviation \\
\hline 1 & $29 \%$ & 3.8 \\
\hline 2 & $30 \%$ & 3.3 \\
\hline 3 & $32 \%$ & 2.8 \\
\hline 4 & $72 \%$ & 6.4 \\
\hline 5 & $65 \%$ & 6.3 \\
\hline 6 & $68 \%$ & 2.7 \\
\hline 7 & $22 \%$ & 3.1 \\
\hline 8 & $59 \%$ & 3.2 \\
\hline
\end{tabular}




\subsection{Impact Of Implementing The Revised Nurse-Patient Ratio}

To study the impact of implementing the revised nurse-patient ratio, we considered that patients in rooms 2 and 3 (total of seven EGD and colonoscopy patients) would undergo MAC in addition to patients scheduled for ERCP and EUS. Table 6 summarizes the revised statistics. Note that although patient flow time is relatively unchanged, the overtime is substantially increased $(50 \%)$ as more recovery nurses' time is needed after 5:00 P.M.

Table 6: Key Statistics for the baseline model with revised nurse-patient ratio

\begin{tabular}{|l|c|c|}
\hline \multicolumn{1}{|c|}{ Measurement } & Average & Std Deviation \\
\hline Patient Flow Time (Minutes) & 177.8 & 7.0 \\
\hline Waiting for Procedure Room (Minutes) & 31.5 & 0.5 \\
\hline Waiting for Recovery Nurse (Minutes) & 0.0 & 0.0 \\
\hline Overtime (hours) & 5.1 & 0.5 \\
\hline
\end{tabular}

\section{STRATEGIES TO IMPROVE UNIT EFFICIENCY IMPACTED BY NURSE-PATIENT RATIO}

Several strategies were considered for improving unit efficiency impacted by the nurse-patient ratio. These strategies can be clustered into two groups: increasing nursing support in recovery and smoothing demand in recovery.

\subsection{Increase Nursing Support In Recovery}

Once the first set of patients of the day are prepared, the demand for the preparation bays drops. In addition, the demand for the preparation is incrementally reduced further as the last patient of each room is prepared. Thus, it seemed reasonable to shift a preparation nurse to recovery when the demand for nurses in recovery is at its peak. A potential consequence of this decision that needs to be considered is that a procedure room may become starved for patients because patients are not yet prepared when a procedure room becomes available.

Alternatively, an additional full time or part time recovery nurse could be assigned to the unit. As would be expected in this scenario, the nurse-patient ratio may no longer be binding and the new binding constraint for recovery becomes the number of recovery bays.

\subsection{Smoothing Demand In Recovery}

As mentioned, patients who undergo MAC for complex EGD, colonoscopy, EUS and ERCP procedures require more recovery nurses because the nurse-patient ratio for these patients is 1 to 2 . Furthermore, EUS and ERCP procedures are also longer procedures and patients who undergo these procedures have a longer recovery time; in fact, most often, these are the patients who are still in the unit during overtime hours. EGD and bronchoscopy procedures are shorter than the others, although bronchoscopy patients have a longer recovery time.

Considering these variations, there is an opportunity to smooth the demand for recovery support by two mechanisms. First, a staggered appointment scheme where patients arrive at different times for each 
procedure type may be beneficial. Secondly, considering that the number of available preparation nurses are less than the number of patients who are scheduled early in the morning, patients with the shortest procedure time could be prepared first so that they begin to use the recovery capacity early thus flattening the peak demand.

In the next section, we discuss the results from examining these strategies.

\section{SIMULATION RESULTS}

Table 7 summarizes the output statistics for 10 experiments that reflect the strategies discussed above. For all scenarios, the model is run for a day and for 100 replications. The first scenario is the baseline model discussed earlier in section 4.2, namely 4 preparation and 4 recovery nurses with a nurse-patient ratio of 1 to 3 for all procedures. The second scenario is the repeat of the scenario discussed in section 4.3, namely 4 preparation and 4 recovery nurses with a nurse-patient ratio of 1 to 3 for nurse-sedated patients and 1 to 2 for MAC patients. When this policy is implemented, the model predicted a $50 \%$ increase in nurse overtime. This scenario serves as the baseline for comparing other strategies

\subsection{Smoothing Demand In Recovery}

Scenarios 3 and 4 address the question of which patients should be prepared first in the morning by the four preparation nurses available. In the first scenario, MAC patients are prepared first (three MAC patients). In the second scenario, sedation patients are prepared first (three sedation patients). Recall that since rooms 4 and 5 are assigned to a single physician, there was no need to prepare both patients at the same time. The model did not show a statistical difference between these two scenarios nor did they differ from the revised baseline.

Table 7: Summary Results of Simulation Model

\begin{tabular}{|c|c|c|c|c|c|c|c|c|c|}
\hline \multirow[t]{2}{*}{ No. } & \multirow[t]{2}{*}{ Scenario } & \multicolumn{2}{|l|}{$b^{5}$} & \multirow{2}{*}{$\frac{\mathrm{s}^{0}}{\text { Average }}$} & \multirow{2}{*}{ 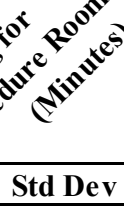 } & \multirow{2}{*}{$\frac{\mid{ }^{0}}{\text { Average }}$} & \multirow{2}{*}{ 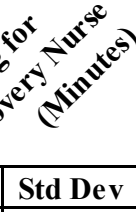 } & \multicolumn{2}{|c|}{$0^{e^{x^{2}}}$} \\
\hline & & Average & Std Dev & & & & & Average & Std Dev \\
\hline 1 & Baseline & 177.8 & 7.0 & 31.4 & 5.1 & 0.0 & 0.0 & 3.4 & 0.4 \\
\hline 2 & Baseline - Revised Ratios & 177.8 & 7.0 & 31.5 & 5.1 & 0.0 & 0.0 & 5.1 & 0.5 \\
\hline 3 & Priority given to MAC & 178.1 & 7.2 & 31.7 & 5.3 & 0.0 & 0.0 & 5.0 & 0.5 \\
\hline 4 & Priority given to Sedation & 176.6 & 6.6 & 30.0 & 4.7 & 0.0 & 0.0 & 5.1 & 0.6 \\
\hline 5 & Staggered MAC & 177.4 & 7.9 & 30.5 & 5.7 & 0.0 & 0.0 & 4.2 & 0.6 \\
\hline 6 & Staggered Sedation & 175.5 & 7.1 & 28.8 & 4.7 & 0.0 & 0.0 & 5.0 & 0.6 \\
\hline 7 & Baseline - 3 Nurses & 178.6 & 7.0 & 32.2 & 5.1 & 0.5 & 0.4 & 5.1 & 0.5 \\
\hline 8 & Baseline - Part Time Nurse & 178.1 & 6.9 & 31.8 & 5.0 & 0.2 & 0.3 & 5.1 & 0.5 \\
\hline 9 & Baseline - Shared Nurse & 178.0 & 7.1 & 31.6 & 5.2 & 0.1 & 0.2 & 5.1 & 0.5 \\
\hline 10 & Staggered MAC wt. Shared Nurse & 177.9 & 7.7 & 30.9 & 5.4 & 0.1 & 0.1 & 4.3 & 0.6 \\
\hline
\end{tabular}

Scenarios 5 and 6 tackle the question of smoothing load by staggering patient scheduling. In the baseline model, the preparation of all patients starts at 7:30am. In scenario 5, MAC patients are scheduled to start preparation at 7:00am, while nurse-sedation patients are kept as they are. In scenario 6, this is reversed (i.e. sedation patients are scheduled to start preparation at 7:00am, while anesthesia patients are kept as they are). In both cases, the total number of nurse-hours required to complete the scheduled load is equal to the total number of nurse-hours in the baseline model. Since MAC patients are more 
commonly treated during overtime, starting these patients 30 minutes early reduces the number of MAC patients in recovery during overtime hours thus substantially reducing nurse overtime ( $18 \%)$.

\subsection{Increase Nursing Support In Recovery}

To explore the need for increased nursing support in recovery, we first studied the impact of reducing nurse support in recovery by one nurse (scenario 7). In this scenario, the waiting time for recovery increased slightly, although this change did not have a significant impact on patient flow time or nurse overtime.

The finding in scenario 7 suggests that the endoscopy unit has an oversupply of nursing staff in recovery and may operate similarly with one less full time recovery nurse. In this context, the next two scenarios address the impact of altering nursing support in recovery. In scenario 8, we demonstrate the impact of replacing a full-time recovery nurse with a part time nurse during the peak demand time for four hours; in this scenario, operational metrics were not significantly different from baseline. Scenario 9 addresses the question of sharing nursing resources between preparation and recovery in the context of a reduction in recovery nurse staffing to three. In this scenario, rather than hiring a part time recovery nurse, a preparation nurse is moved to recovery for an hour in the morning and for 2.5 hours in the afternoon as demand for preparation drops. The results again were unchanged from baseline, suggesting that sharing a pool of preparation and recovery nurses could potentially provide savings. It also suggests that in designing new units, consideration should be given to designs that facilitates sharing resources.

Finally, scenario 10 combines smoothing with sharing. Specifically, we assume three full-time recovery nurses with a fourth nurse providing support during peak hours as a shared resource from the preparation area. In addition, the MAC procedures are scheduled earlier than nurse-sedation cases in a staggered fashion. This option provides opportunity for labor saving as well as scheduling flexibility to the nursing staff and was the preferred scenario among unit leadership.

\section{CONCLUSION}

Improved efficiency of healthcare delivery is a critical goal for large health care systems adapting to health care reform. The application of systems engineering tools, such as discrete event simulation, to health care practice has the potential to provide innovative solutions to very complex problems. In this paper, we describe our efforts partnering with Duke University Medical Center to create a discrete event simulation model of a hospital endoscopy unit and review strategies to improve unit efficiency and reduce nurse overtime. The results suggest that the endoscopy unit could decrease the risk of nurse overtime by staggering the scheduling of MAC patients. After reviewing the results of our analysis, the nursing team at Duke elected to proceed with the staggered patient schedule and shared recovery nurse model. These changes have been adopted and the resulting effects on unit operations are currently being evaluated.

In addition to assisting in planning of the endoscopy unit at Duke University Medical Center, our study also provides generalizable knowledge regarding the operations of hospital endoscopy units. For example, our results highlight the importance of designing endoscopy units that allow shared nursing resources, specifically with shifting work responsibilities between prep and recovery nurses during peak demand for recovery. In addition, the study highlights an opportunity for developing an optimization scheme for outpatient endoscopy scheduling; this challenge is the focus of ongoing work. 
Taheri, Gellad, Burchfield, and Cooper

\section{REFERENCES}

Berg B, B. Denton, H. Nelson, H. Balasubramanian, A. Rahman , A. Bailey, and K. Lindor. 2010. “A Discrete Event Simulation Model to Evaluate Operational Performance of a Colonoscopy Suite." Medical Decision Making 30(3): 380-387.

Centeno M.A., M. Dodd, M. Aranda, and J. Sanchez. 2006. "A Simulation Study To Increase Throughput In An Endoscopy Center." Proceedings of the 2010 Winter Simulation Conference 2462-2473.

Deas, T.M., "Health-care value-based purchasing." Gastrointest Endosc Clin N Am. 16(4): p.643-56.

Gellad Z.F., and D. Provenzale. 2010. "Colorectal cancer: national and international perspective on the burden of disease and public health impact." Gastroenterology 138:2177-90.

Harewood, G.C. and D. Lieberman, 2004. "Colonoscopy practice patterns since introduction of Medicare coverage for average-risk screening." Clin. Gastroenterol. Hepatol. 2: p. 72-77.

Hewett, D.G. and D.K. Rex, 2010. "Improving Colonoscopy Quality Through Health-Care Payment Reform." Am J Gastroenterol 105(9): p. 1925-1933.

Kelton W.D, R.P. Sadowski, D.T. Sturrock, and N.B. Swets. 2009. Simulation With Arena, McGraw-Hill Higher Education .

Kelton W.D., J.S. Smith, D.T. Sturrock, and V. Verbraeck. 2010. Simio \& Simulation, McGraw-Hill Higher Education.

Kim, L.S. 2011. "How Will Accreditation of Your Ambulatory Endoscopy Center Be an Essential Component of Showing Value-Based Health Care?" Clin Gastro Hep 9(1): p. 21-23.

Lieberman, D.A., J.E. Holub, D. Kraemer, and C.D. Morris. 2005. "Utilization of colonoscopy in the United States: results from a national consortium." Gastrointest Endoscopy. 62: p. 875-883.

Building a Better Delivery System: a new engineering/health care partnership, in Committee on Engineering and the Health Care System; edited by Reid, P.P., W.D. Compton, J.H. Grossman, and G. Fanjiang, 2005. National Academy of Engineering and Institute of Medicine. National Academy Press: Washington, DC.

Rex D.K., B.J. Lahue, R.W. Dronzek, and M.J. Lacey. 2005. "Impact of Two Procedure Rooms Per Physician on Productivity: Computer Simulation Examines the Impact of Process Change in the Hospital Gasroenterology Department." Gastrointestinal Endoscopy. 61:AB154-AB.

Schenck A.P., S.C. Peacock, C.N. Klabunde, P. Lapin, J.F. Coan, and M.L. Brown., 2009. "Trends in Colorectal Cancer Test Use in the Medicare Population, 1998-2005." American Journal of Preventive Medicine. 37(1): p. 1-7.

Seeff L.C., D.L. Manninen, F.B. Dong, S.K. Chattopadhyay, M.R. Nadel, F.K. Tangka, and N.A. Molinaril. 2004. "Is there endoscopic capacity to provide colorectal cancer screening to the unscreened population in the United States?" Gastroenterology. 127(6): p. 1661.

U.S. Department of Health and Human Services Report to Congress: Medicare Ambulatory Surgical Center Value-Based Purchasing Implementation Plan, accessed 11/3/2011 at https://www.cms.gov/ASCPayment/ 
Taheri, Gellad, Burchfield, and Cooper

\section{AUTHOR BIOGRAPHIES}

JAVAD TAHERI, PhD is a Research Associate Professor in the Edward P. Fitts Department of Industrial and Systems Engineering of North Carolina State University. Since 2009, Dr. Taheri has had a joint appointment with Durham VA Medical Center as a Systems Redesign specialist where he has employed Industrial and Systems Engineering methodologies to improve patient flows and operational efficiencies in several clinics. Dr. Taheri is also the project manager for the NC Health Alert Network research project, a public health emergency preparedness research project funded through the CDC NC PERRC. Prior to joining NC State, Dr. Taheri worked for Nortel from 1984-2002 where he was the Director of Operations Research from 1994 to 2002. His research and teaching interests are in the areas of business process modeling and simulation, patient flow analysis, scheduling, and decision support tool development.

ZIAD GELLAD, MD MPH is an assistant professor of medicine at the Duke University School of Medicine and a faculty member of the Duke Clinical Research Institute. He also holds an appointment in the Center for Health Services Research at the Durham VA Medical Center where he is a staff physician in Gastroenterology. Dr. Gellad received his MD degree from the Johns Hopkins University School of Medicine and his MPH from the Johns Hopkins Bloomberg School of Public Health. He completed a residency in internal medicine and a fellowship in gastroenterology at Duke University Medical Center. His research interests include health services research in quality of care and optimizing health care value. He was recently awarded a career development award from the American Society for Gastrointestinal Endoscopy for his work studying physician practice variation in colorectal cancer screening. He can be reached atziad.gellad@duke.edu

DARIELE BURCHFIELD, RN, CGRN is certified in Gastroenterology who has dedicated her 17 year nursing career to the GI Endoscopy/Bronchoscopy Unit at Duke University Medical Center (DUMC). She is currently Nurse Manager for the Endoscopy Unit at Duke University Medical Center. Performance improvement credentials include achievement of Six Sigma Green Belt, Change Management training, LEAN training, leader of six sigma process improvement for the Endoscopy/Bronchoscopy health system, and leader for the Patient Satisfaction Initiative for DUMC. Dariele's experiences and responsibilities include Strategic Service Associate, Nurse Management of the DUMC Endoscopy/Bronchoscopy Unit, advisor for the Clinical Ladder Program for the entire Duke University Health System, cocoordinator for the Endoscopic Ultrasound/ERCP Course at DUMC, Faculty for the ASGE Safety and Quality Course, and Nurse Consultant for operational efficiency.

KEVIN COOPER is a Director in the Performance Services department at Duke University Hospital. He has been with Duke for approximately 10 years and has served in several different capacities. He has supported multiple inpatient clinical service units, emergency services, diagnostic services as well as the ambulatory care division and the Patient Revenue Management Organization with performance improvement strategies. Kevin is also a certified black belt in the Six Sigma problem solving methodology. He played a significant role in the development and implementation of the Hospital's Six Sigma program. Prior to joining Duke, Kevin worked as a consultant for over 6 years with Cap Gemini Ernst \& Young and Premier Inc. in their health care consulting practice. His major competency groups included business transformation management, subject matter specialist, project management and community based management engineer. He has led substantial reengineering and cost reduction projects to include labor assessment and restructuring as well as hospital layout and departmental redesign. Kevin Cooper holds a Bachelor of Science degree in Industrial Engineering and a minor in Economics from North Carolina State University. 